\title{
Neutrino oscillations beyond two flavours
}

\author{
E. Kh. Akhmedova * \\ ${ }^{a}$ Centro de Física das Interacções Fundamentais \\ Departamento de Física, Instituto Superior Técnico \\ Av. Rovisco Pais, P-1049-001 Lisboa, Portugal
}

I review some theoretical aspects of neutrino oscillations in the case when more than two neutrino flavours are involved. These include: approximate analytic solutions for 3-flavour (3f) oscillations in matter; matter effects in $\nu_{\mu} \leftrightarrow \nu_{\tau}$ oscillations; 3f effects in oscillations of solar, atmospheric, reactor and supernova neutrinos and in accelerator long-baseline experiments; $\mathrm{CP}$ and $\mathrm{T}$ violation in neutrino oscillations in vacuum and in matter; the problem of $U_{e 3} ;$ if oscillations.

\section{INTRODUCTION}

Explanation of the solar and atmospheric neutrino data in terms of neutrino oscillations requires at least three neutrino species, and in fact three neutrino species are known to exist $-\nu_{e}, \nu_{\mu}$ and $\nu_{\tau}$. If the LSND experiment is correct, then probably a fourth neutrino type should exist, a light sterile neutrino $\nu_{s}$. However, until relatively recently most of the studies of neutrino oscillations were performed in the 2-flavour framework. There were essentially two reasons for that: (1) simplicity - there are much fewer parameters in the 2-flavour case than in the 3-flavour one, and the expressions for the transition probabilities are much simpler and by far more tractable, and (2) the hierarchy of $\Delta m^{2}$ values, which allows to effectively decouple different oscillation channels. The 2-flavour approach proved to be a good first approximation, which is a consequence of the hierarchy $\Delta m_{\odot} \ll \Delta m_{\text {atm }}$ and of the smallness of the leptonic mixing parameter $\left|U_{e 3}\right|$.

However, the increased accuracy of the available and especially forthcoming neutrino data makes it very important to take into account even relatively small effects in neutrino oscillations. In addition, the experimentally favoured solution of the solar neutrino problem is at present the LMA

*On leave from National Research Centre Kurchatov Institute, Moscow, Russia. Supported by the Calouste Gulbenkian Foundation as a Gulbenkian Visiting Professor.
MSW one, which requires the hierarchy between $\Delta m_{\odot}$ and $\Delta m_{\text {atm }}$ to be relatively mild. Also, effects specific to $\geq 3$ flavour neutrino oscillations, such as $\mathrm{CP}$ and $\mathrm{T}$ violation, are now being very widely discussed. All this makes 3-flavour (or 4flavour) analyses of neutrino oscillations mandatory.

In my talk I review some theoretical issues pertaining to neutrino oscillations in the case when more than two neutrino species are involved. I mainly concentrate on 3-flavour (3f) oscillations and only very briefly consider the $4 \mathrm{f}$ case. The topics that are discussed include: approximate analytic solutions for $3 \mathrm{f}$ oscillations in matter; matter effects in $\nu_{\mu} \leftrightarrow \nu_{\tau}$ oscillations; 3f effects in oscillations of solar, atmospheric, reactor and supernova neutrinos and in accelerator longbaseline experiments; $\mathrm{CP}$ and $\mathrm{T}$ violation in neutrino oscillations in vacuum and in matter; the problem of $U_{e 3} ;$ ff oscillations.

\section{NEUTRINO OSCILLATIONS IN MATTER (3f)}

Neutrino oscillations in matter are described by the evolution equation $i(d / d t) \nu=H \nu$, where $\nu=\left(\begin{array}{lll}\nu_{e} & \nu_{\mu} & \nu_{\tau}\end{array}\right)^{T}$ and

$H=\left[U\left(\begin{array}{ccc}E_{1} & 0 & 0 \\ 0 & E_{2} & 0 \\ 0 & 0 & E_{3}\end{array}\right) U^{\dagger}+\left(\begin{array}{ccc}V(t) & 0 & 0 \\ 0 & 0 & 0 \\ 0 & 0 & 0\end{array}\right)\right]$ 


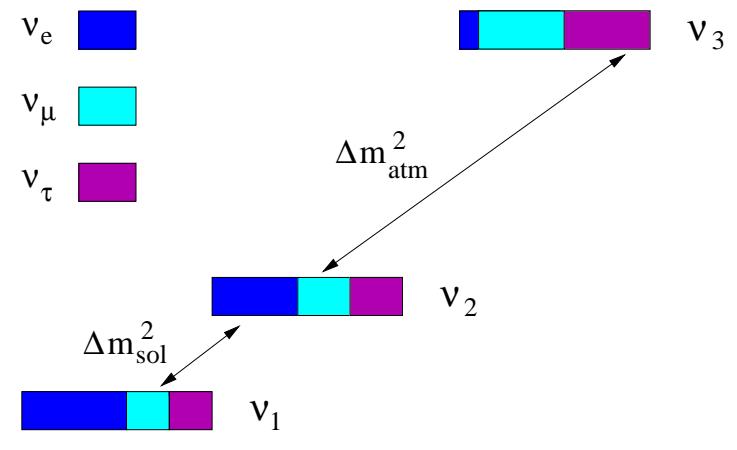

Figure 1. Normal mass hierarchy



Figure 2. Inverted mass hierarchy

The effective potential $V=\sqrt{2} G_{F} N_{e}$ is due to the charged-current interaction of $\nu_{e}$ with the electrons of the medium. The neutral current induced potentials are omitted from Eq. (11) because they are the same for neutrinos of all three species and therefore do not affect neutrino oscillations. This, however, is only true in leading (tree) order; radiative corrections induce tiny differences between the neutral current potentials of $\nu_{e}, \nu_{\mu}$ and $\nu_{\tau}$ and, in particular, result in a very small $\nu_{\mu}-\nu_{\tau}$ potential difference $V_{\mu \tau} \sim 10^{-5} V$ [1]. This quantity is negligible in most situations but may be important for supernova neutrinos.

For matter of constant density, closed-form solutions of the evolution equation can be found [2]; however, the corresponding expressions are rather complicated and not easily tractable. For a general electron density profile $N_{e} \neq$ const no closed-form solutions exist. It is therefore desirable to have approximate analytic solutions of the neutrino evolution equation. A number of such solutions were found, most of them based on the expansions in one (or both) of the two small parameters:

$$
\begin{aligned}
& \Delta m_{21}^{2} / \Delta m_{31}^{2}=\Delta m_{\odot}^{2} / \Delta m_{\mathrm{atm}}^{2} \lesssim 0.1, \\
& \left|U_{e 3}\right|=\left|\sin \theta_{13}\right| \lesssim 0.2[3] .
\end{aligned}
$$

Our numbering of neutrino mass eigenstates corresponds to that in Figs. 1 and 2, which also show schematically the possible neutrino mass hierarchies and the flavour composition of neutrino mass eigenstates.

In the limits $\Delta m_{21}^{2}=0$ or $U_{13}=0$ the transition probabilities acquire an effective $2 \mathrm{f}$ form. When both these parameters vanish, the genuine $2 \mathrm{f}$ case is recovered.

\subsection{Constant-density matter}

In the case of matter of constant density approximate solutions of the neutrino evolution equation were found using the expansion in $\alpha \equiv$ $\Delta m_{\odot}^{2} / \Delta m_{\text {atm }}^{2}$ in $[$. An expansion in both $\alpha$ and $\sin \theta_{13}$ was used in [5]. The $\nu_{e} \leftrightarrow \nu_{\mu}$ transition probability found in [5] has the general form

$$
\begin{aligned}
P\left(\nu_{e} \leftrightarrow \nu_{\mu}\right) \sim s_{23}^{2} \tilde{P}_{2}\left(\Delta m_{31}^{2}, \theta_{13}, N_{e}\right)+ \\
\\
c_{23}^{2} \tilde{P}_{2}\left(\Delta m_{21}^{2}, \theta_{12}, N_{e}\right)+\text { interf. term },
\end{aligned}
$$

where the quantities $\tilde{P}_{2}$ are the $2 \mathrm{f}$ transition probabilities in matter depending on the corresponding parameters shown in the parentheses. The interference term, which is linear in both $\alpha$ and $\sin \theta_{13}$, describes the genuine $3 \mathrm{f}$ effects, both CPconserving and $\mathrm{CP}$-violating.

\subsection{Arbitrary density profile}

Matter of constant density is a good first approximation for long-baseline accelerator neutrino experiments (neutrinos traverse the mantle of the Earth). However, it is not very useful for describing the oscillations of solar, atmospheric and supernova neutrinos. An alternative approach is to consider matter with an arbitrary density profile and reduce the problem to an effective 2 f one plus easily calculable 3 f corrections. 


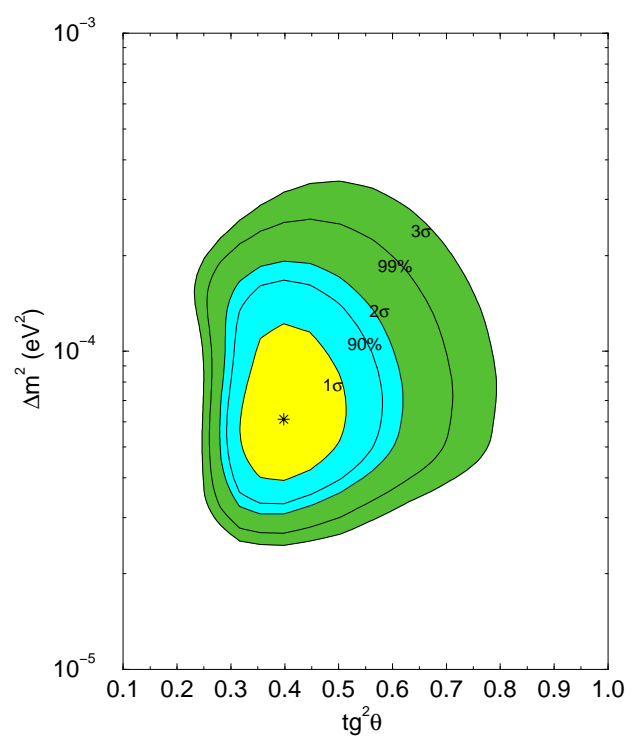

Figure 3. LMA allowed parameter region for $\theta_{13}=0$ 12

This has been done using the expansion in $\alpha$ in [6] and the expansion in $\sin \theta_{13}$ in [7 9]. A different approach, based on the adiabatic approximation, was employed, e.g., in 10].

\subsection{Matter effects in $\nu_{\mu} \leftrightarrow \nu_{\tau}$ oscillations}

Since the matter-induced potentials for $\nu_{\mu}$ and $\nu_{\tau}$ are the same (neglecting the radiative corrections), in the $2 \mathrm{f}$ case the $\nu_{\mu} \leftrightarrow \nu_{\tau}$ oscillations are not affected by matter. This, however, is not true in the $3 \mathrm{f}$ case; therefore matter effects on $\nu_{\mu} \leftrightarrow \nu_{\tau}$ oscillations is a pure $3 \mathrm{f}$ effect. It vanishes only when both $\Delta m_{21}^{2}$ and $U_{e 3}$ vanish.

\section{3f EFFECTS IN $\nu$ OSCILLATIONS}

We shall now discuss $3 \mathrm{f}$ effects in oscillations of neutrinos from various sources.

\subsection{Solar neutrinos}

In the $3 \mathrm{f}$ case, solar $\nu_{e}$ can in principle oscillate into either $\nu_{\mu}$, or $\nu_{\tau}$, or some their combination. What do they actually oscillate to?

It is easy to answer this question. The smallness of the mixing parameter $\left|U_{e 3}\right|$ implies that the mass eigenstate $\nu_{3}$ is approximately given by

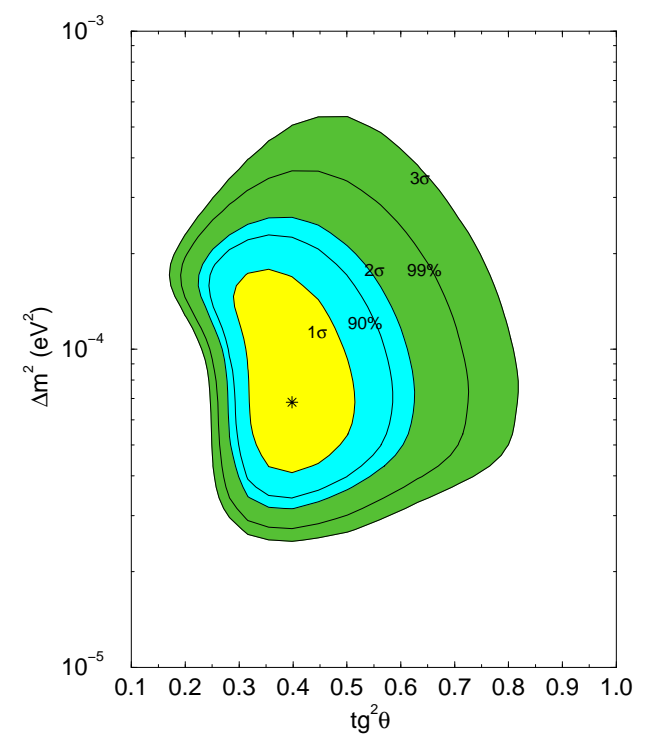

Figure 4. LMA allowed parameter region for $\sin ^{2} \theta_{13}=0.04$ 12]

$\nu_{3} \simeq s_{23} \nu_{\mu}+c_{23} \nu_{\tau}$

and, to first approximation, does not participate in the solar neutrino oscillations. From the unitarity of the leptonic mixing matrix it then follows that the solar neutrino oscillations are the oscillations between $\nu_{e}$ and a state $\nu^{\prime}$ which is the linear combination of $\nu_{\mu}$ and $\nu_{\tau}$, orthogonal to $\nu_{3}$ :

$\nu^{\prime}=c_{23} \nu_{\mu}-s_{23} \nu_{\tau}$

Since the mixing angle $\theta_{23}$, responsible for the atmospheric neutrino oscillations, is known to be close to $45^{\circ}$, Eq. (6) implies that the solar $\nu_{e}$ oscillate into a superposition of $\nu_{\mu}$ and $\nu_{\tau}$ with equal or almost equal weights.

What are the $3 \mathrm{f}$ effects in the oscillation probabilities? Since at low energies $\nu_{\mu}$ and $\nu_{\tau}$ are experimentally indistinguishable, all the observables depend on just one probability - the $\nu_{e}$ survival probability $P\left(\nu_{e} \rightarrow \nu_{e}\right)$. Averaging over fast oscillations due to the large mass squared difference $\Delta m_{\mathrm{atm}}^{2}=\Delta m_{31}^{2}$ yields [1]

$P\left(\nu_{e} \rightarrow \nu_{e}\right) \simeq c_{13}^{4} \tilde{P}_{2 e e}\left(\Delta m_{21}^{2}, \theta_{12}, N_{\text {eff }}\right)+s_{13}^{4}$.

Here $\tilde{P}_{2 e e}\left(\Delta m_{21}^{2}, \theta_{12}, N_{\text {eff }}\right)$ is the 2 f survival probability of $\nu_{e}$ in matter with the effective electron 
density $N_{\text {eff }}=c_{13}^{2} N_{e}$.

As follows from the CHOOZ data [3], the second term in Eq. (77), $s_{13}^{4}$, does not exceed $10^{-3}$, i.e. is negligible. At the same time, the coefficient $c_{13}^{4}$ of $\tilde{P}_{2 e e}$ in the first term may differ from unity by as much as $\sim 5-10 \%$. Thus, 3 f effects may lead to an energy-independent suppression of the $\nu_{e}$ survival probability by up to $10 \%$. With high precision solar data this must be taken into account. This is illustrated by Figs. 3 and 1 [12]: The difference between the cases $\theta_{13}=0$ and $\sin ^{2} \theta_{13}=0.04$ (which is about the maximum allowed by $\mathrm{CHOOZ}$ value) is quite noticeable.

\subsection{Atmospheric neutrinos}

(1) The dominant channel $\nu_{\mu} \leftrightarrow \nu_{\tau}$. In the $2 \mathrm{f}$ limit, there are no matter effects in this channel (neglecting tiny $V_{\mu \tau}$ caused by radiative corrections). The oscillation probability is independent from the sign of $\Delta m_{31}^{2}$, i.e. cannot differentiate between the normal and inverted neutrino mass hierarchies. The $3 \mathrm{f}$ effects result in a weak sensitivity to matter effects and to the sign of $\Delta m_{31}^{2}$.

(2) The subdominant channels $\nu_{e} \leftrightarrow \nu_{\mu, \tau}$. Contributions of these oscillation channels to the number of $\mu$ - like events are subleading and difficult to observe. For e-like events, one could $a$ priori expect significant oscillations effects. However, these effects are in fact strongly suppressed because of the specific composition of the atmospheric neutrino flux and proximity of the mixing angle $\theta_{23}$ to $45^{\circ}$. Indeed, in the $2 \mathrm{f}$ limits one finds

$$
\frac{F_{e}-F_{e}^{0}}{F_{e}^{0}}=\tilde{P}_{2}\left(\Delta m_{31}^{2}, \theta_{13}, V\right) \cdot\left(r s_{23}^{2}-1\right)
$$

in the limit $\Delta m_{21}^{2} \rightarrow 0$ [6], and

$$
\frac{F_{e}-F_{e}^{0}}{F_{e}^{0}}=\tilde{P}_{2}\left(\Delta m_{21}^{2}, \theta_{12}, V\right) \cdot\left(r c_{23}^{2}-1\right)
$$

in the limit $s_{13} \rightarrow 0$ []. Here $F_{e}^{0}$ and $F_{e}$ are the $\nu_{e}$ fluxes in the absence and in the presence of the oscillations, respectively, and $r \equiv F_{\mu}^{0} / F_{e}^{0}$. At low energies $r \simeq 2$; also, we know that $s_{23}^{2} \simeq$ $c_{23}^{2} \simeq 1 / 2$. Therefore the factors $\left(r s_{23}^{2}-1\right)$ and $\left(r c_{23}^{2}-1\right)$ in Eqs. (8) and (9) are very small and strongly suppress the oscillation effects even if the transition probabilities $\tilde{P}_{2}$ are close to unity. This happens because of the strong cancellations of the transitions from and to the $\nu_{e}$ state.

All this looks as a conspiracy to hide the oscillation effects on the e-like events! This conspiracy is, however, broken by the $3 \mathrm{f}$ effects. Keeping both $\Delta m_{21}^{2}$ and $s_{13}$ in leading order yields [9]

$$
\begin{aligned}
\frac{F_{e}-F_{e}^{0}}{F_{e}^{0}} & \simeq \tilde{P}_{2}\left(\Delta m_{31}^{2}, \theta_{13}\right) \cdot\left(r s_{23}^{2}-1\right) \\
& +\tilde{P}_{2}\left(\Delta m_{21}^{2}, \theta_{12}\right) \cdot\left(r c_{23}^{2}-1\right) \\
& -2 s_{13} s_{23} c_{23} r \operatorname{Re}\left(\tilde{A}_{e e}^{*} \tilde{A}_{\mu e}\right)
\end{aligned}
$$

The interference term, which represents the genuinely $3 \mathrm{f}$ effects, is not suppressed by the flavour composition of the atmospheric neutrino flux; it may be responsible (at least, partially) for some excess of the upward-going sub-GeV e-like events observed at Super-Kamiokande [9].

\subsection{Reactor antineutrinos}

Since the average energy of reactor $\bar{\nu}_{e}$ 's is $\bar{E} \sim 3$ $\mathrm{MeV}$, for intermediate-baseline experiments, such as $\mathrm{CHOOZ}$ and Palo Verde $(L \sim 1 \mathrm{~km})$, one has

$\frac{\Delta m_{31}^{2}}{4 E} L \sim 1, \quad \frac{\Delta m_{21}^{2}}{4 E} L \ll 1$.

This justifies the use of the one mass scale dominance approximation, which gives

$P\left(\bar{\nu}_{e} \rightarrow \bar{\nu}_{e}\right)=1-\sin ^{2} 2 \theta_{13} \cdot \sin ^{2}\left(\frac{\Delta m_{31}^{2}}{4 E} L\right)$,

a pure $2 \mathrm{f}$ result. However, in the case of the LMA solution of the solar neutrino problem, at high enough confidence level $\Delta m_{21}^{2}$ can be comparable with $\Delta m_{31}^{2}$, and the second condition in (12) may not be valid. In such a situation the $3 \mathrm{f}$ effects coming through the subdominant $\Delta m_{21}^{2}$ should be taken into account, The analyses 13 show that the constraints on $\left|U_{e 3}\right|$ derived from the CHOOZ experiment become slightly more stringent in that case. However, the new SNO data [14] disfavour large values of $\Delta m_{21}^{2}$ and so make this possibility less likely.

For KamLAND, which is a very long baseline reactor experiment $(\bar{L} \simeq 170 \mathrm{~km})$, one has

$$
\frac{\Delta m_{31}^{2}}{4 E} L \gg 1, \quad \frac{\Delta m_{21}^{2}}{4 E} L \gtrsim 1 \quad \text { (for LMA) . }
$$


Averaging over the fast oscillations driven by $\Delta m_{31}^{2}=\Delta m_{\text {atm }}^{2}$ yields

$P\left(\bar{\nu}_{e} \rightarrow \bar{\nu}_{e}\right)=c_{13}^{4} P_{2 \bar{e} \bar{e}}\left(\Delta m_{21}^{2}, \theta_{12}\right)+s_{13}^{4}$.

This has the same form as Eq. (7), except that the $2 \mathrm{f}$ survival probability $P_{2 \bar{e} \bar{e}}$ has to be calculated in vacuum rather than in matter; it is in fact given by Eq. (13). The probability (15) can differ from the 2 f probability (13) by up to $\sim 10 \%$.

\subsection{LBL accelerator experiments}

(1) $\nu_{\mu}$ disappearance.

$3 \mathrm{f}$ effects can result in up to $\sim 10 \%$ corrections to the disappearance probability, mainly due to the factor $c_{13}^{4}$ in the effective amplitude of the $\nu_{\mu} \leftrightarrow \nu_{\tau}$ oscillations,

$$
\sin ^{2}\left(2 \theta_{\mu \tau}\right)_{\mathrm{eff}}=c_{13}^{4} \sin ^{2} 2 \theta_{23} .
$$

Another manifestation of 3-flavourness are small matter effects in $\nu_{\mu} \leftrightarrow \nu_{\tau}$ oscillations. The same applies to $\nu_{\tau}$ appearance in experiments with the conventional neutrino beams. $\nu_{\mu}$ disappearance also receives contributions from the subdominant $\nu_{\mu} \leftrightarrow \nu_{e}$ oscillations.

(2) $\nu_{\mu}$ appearance at neutrino factories; $\nu_{e}$ appearance at neutrino factories and in experiments with the conventional neutrino beams.

These are driven by the $\nu_{e} \leftrightarrow \nu_{\mu, \tau}$ oscillations. There are two channels through which these subdominant oscillations can proceed - those governed by the parameters $\left(\theta_{13}, \Delta m_{31}^{2}\right)$ and $\left(\theta_{12}, \Delta m_{21}^{2}\right)$. For typical energies of the LBL accelerator experiments (a few $\mathrm{GeV}$ to tens of $\mathrm{GeV}$ ), and assuming the LMA solution of the solar neutrino problem, one finds that for $\theta_{13}$ in the range $3 \cdot 10^{-3} \lesssim \theta_{13} \lesssim 3 \cdot 10^{-2}$ the two channels compete; otherwise one of them dominates.

Unlike in the case of atmospheric neutrinos, there is no suppression of the oscillation effects on the $\nu_{e}$ flux due to the flavour composition of the original flux.

The dependence of the oscillation probabilities on the CP-violating phase $\delta_{\mathrm{CP}}$ (both $\sim \sin \delta_{\mathrm{CP}}$ and $\sim \cos \delta_{\mathrm{CP}}$ ) comes from the interference terms and is a pure $3 \mathrm{f}$ effect. The $3 \mathrm{f}$ effects will be especially important for the future experiments at neutrino factories which are designed for precision measurements of neutrino parameters.

\subsection{Supernova neutrinos}

In supernovae, matter density varies in a very wide range, and the conditions for the three MSW resonances are satisfied (taking into account that due to radiative corrections $V_{\mu \tau} \neq 0$ ), see Fig. 5 . The hierarchy $\Delta m_{21}^{2} \ll \Delta m_{31}^{2}$ leads to the approximate factorization of transition dynamics at the resonances, so that the transitions, to first approximation, are effectively $2 \mathrm{f}$ ones. However, the observable effects of the supernova neutrino oscillations depend on the transitions between all three neutrino species.

The Earth matter effects on supernova neutrinos can be used to measure $\left|U_{e 3}\right|$ to a very high accuracy $\left(\sim 10^{-3}\right)$ and to determine the sign of $\Delta m_{31}^{2}$ 15.

The transitions due to the $\nu_{\mu}-\nu_{\tau}$ potential difference $V_{\mu \tau}$ caused by radiative corrections may have observable consequences if the originally produced $\nu_{\mu}$ and $\nu_{\tau}$ fluxes are not exactly the same [16].

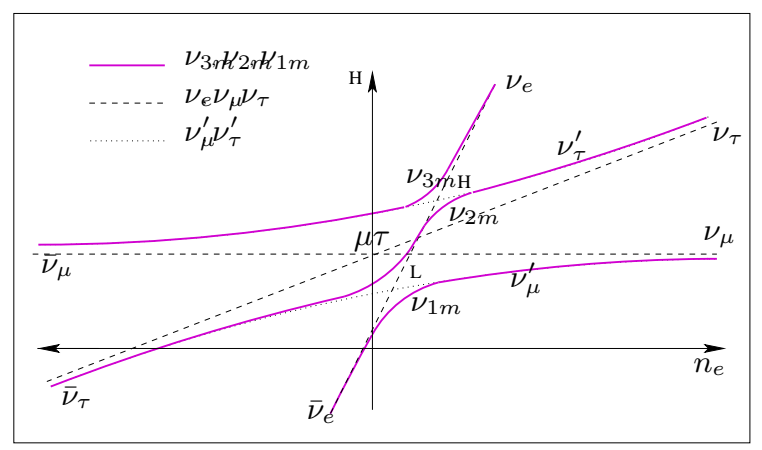

Figure 5. Energy level crossing scheme for supernova neutrinos

\section{CP AND T VIOLATION IN $\nu$ OSCILLATIONS IN VACUUM}

The probability of $\nu_{a} \rightarrow \nu_{b}$ oscillations in vacuum is given by

$P\left(\nu_{a}, t_{0} \rightarrow \nu_{b} ; t\right)=\left|\sum_{i} U_{b i} e^{-i E_{i}\left(t-t_{0}\right)} U_{a i}^{*}\right|^{2}$. 
In the general case of $n$ flavours the leptonic mixing matrix $U_{a i}$ depends on $(n-1)(n-2) / 2$ Diractype $\mathrm{CP}$-violating phases $\left\{\delta_{\mathrm{CP}}\right\}$. If neutrinos are Majorana particles, there are $n-1$ additional, socalled Majorana-type CP-violating phases. However, they do not affect neutrino oscillations and therefore I shall not discuss them.

Under CP transformation, neutrinos are replaced by their antiparticles $\left(\nu_{a, b} \leftrightarrow \bar{\nu}_{a, b}\right)$, which is equivalent to the complex conjugation of $U_{a i}$ :

$$
\begin{aligned}
\mathrm{CP}: & \nu_{a, b} \leftrightarrow \bar{\nu}_{a, b} \\
& \Leftrightarrow U_{a i} \rightarrow U_{a i}^{*} \quad\left(\left\{\delta_{\mathrm{CP}}\right\} \rightarrow-\left\{\delta_{\mathrm{CP}}\right\}\right) .
\end{aligned}
$$

Time reversal transformation interchanges the initial and final evolution times $t_{0}$ and $t$ in Eq. (17), i.e. corresponds to evolution "backwards in time". As follows from Eq. (17), the interchange $t_{0} \rightleftarrows t$ is equivalent to the complex conjugation of the exponential factors in the oscillation amplitude. Since the transition probability only depends on the modulus of the amplitude, this is equivalent to the complex conjugation of the factors $U_{b i}$ and $U_{a i}^{*}$, which in turn amounts to interchanging $a \rightleftarrows b$. Thus, instead of evolution "backwards in time" one can consider evolution forward in time, but between the interchanged initial and final flavours:

$$
\begin{aligned}
T: & t_{0} \rightleftarrows t \Leftrightarrow \nu_{a} \leftrightarrow \nu_{b} \\
& \Rightarrow U_{a i} \rightarrow U_{a i}^{*}\left(\left\{\delta_{\mathrm{CP}}\right\} \rightarrow-\left\{\delta_{\mathrm{CP}}\right\}\right) .
\end{aligned}
$$

Under the combined action of $\mathrm{CP}$ and $\mathrm{T}$ :

$$
\begin{aligned}
\mathrm{CPT}: & \nu_{a, b} \leftrightarrow \bar{\nu}_{a, b} \& t_{0} \rightleftarrows t\left(\nu_{a} \leftrightarrow \nu_{b}\right) \\
& \Rightarrow P\left(\nu_{a} \rightarrow \nu_{b}\right) \rightarrow P\left(\bar{\nu}_{b} \rightarrow \bar{\nu}_{a}\right) .
\end{aligned}
$$

From CPT invariance it follows that $\mathrm{CP}$ violation implies $\mathrm{T}$ violation and vice versa.

$\mathrm{CP}$ and $\mathrm{T}$ violation can be characterized by the probability differences

$$
\begin{aligned}
& \Delta P_{a b}^{\mathrm{CP}} \equiv P\left(\nu_{a} \rightarrow \nu_{b}\right)-P\left(\bar{\nu}_{a} \rightarrow \bar{\nu}_{b}\right), \\
& \Delta P_{a b}^{\mathrm{T}} \equiv P\left(\nu_{a} \rightarrow \nu_{b}\right)-P\left(\nu_{b} \rightarrow \nu_{a}\right) .
\end{aligned}
$$

From CPT invariance it follows that the $\mathrm{CP}$ - and T-violating probability differences coincide, and that the survival probabilities have no $\mathrm{CP}$ asymmetry:

$$
\Delta P_{a b}^{\mathrm{CP}}=\Delta P_{a b}^{\mathrm{T}} ; \quad \Delta P_{a a}^{\mathrm{CP}}=0 .
$$

$\mathrm{CP}$ and $\mathrm{T}$ violations are absent in the $2 \mathrm{f}$ case, so any observable violation of these symmetries in neutrino oscillations in vacuum would be a pure $\geq 3 \mathrm{f}$ effect.

In the $3 \mathrm{f}$ case, there is only one $\mathrm{CP}$-violating Dirac-type phase $\delta_{\mathrm{CP}}$ and so only one CP-odd (and T-odd) probability difference:

$$
\begin{aligned}
& \Delta P_{e \mu}^{\mathrm{CP}}=\Delta P_{\mu \tau}^{\mathrm{CP}}=\Delta P_{\tau e}^{\mathrm{CP}} \equiv \Delta P, \\
& \Delta P=-4 s_{12} c_{12} s_{13} c_{13}^{2} s_{23} c_{23} \sin \delta_{\mathrm{CP}} \times \\
& {\left[\sin \left(\frac{\Delta m_{12}^{2}}{2 E} L\right)+\sin \left(\frac{\Delta m_{23}^{2}}{2 E} L\right)+\sin \left(\frac{\Delta m_{31}^{2}}{2 E} L\right)\right] .}
\end{aligned}
$$

It vanishes

- when at least one $\Delta m_{i j}^{2}=0$

- when at least one $\theta_{i j}=0$ or $90^{\circ}$

- when $\delta_{\mathrm{CP}}=0$ or $180^{\circ}$

- in the averaging regime

- in the limit $L \rightarrow 0$ (as $\left.L^{3}\right)$

Clearly, this quantity is very difficult to observe.

\section{CP AND T VIOLATIONS IN $\nu$ OSCILLATIONS IN MATTER}

For neutrino oscillations in matter, CP transformation (substitution $\nu_{a} \leftrightarrow \bar{\nu}_{a}$ ) implies not only complex conjugating the leptonic mixing matrix, but also flipping the sign of the matter-induced neutrino potentials:

$$
\begin{gathered}
\mathrm{CP}: \quad U_{a i} \rightarrow U_{a i}^{*}\left(\left\{\delta_{\mathrm{CP}}\right\} \rightarrow-\left\{\delta_{\mathrm{CP}}\right\}\right), \\
V(r) \rightarrow-V(r) .
\end{gathered}
$$

It can be shown [8] that in matter with an arbitrary density profile, as well as in vacuum, the action of time reversal on neutrino oscillations is equivalent to interchanging the initial and final neutrino flavours. It is also equivalent to complex conjugating $U_{a i}$ and replacing the matter density profile by the reverse one:

$$
\begin{gathered}
\mathrm{T}: U_{a i} \rightarrow U_{a i}^{*}\left(\left\{\delta_{\mathrm{CP}}\right\} \rightarrow-\left\{\delta_{\mathrm{CP}}\right\}\right), \\
V(r) \rightarrow \tilde{V}(r) .
\end{gathered}
$$

Here

$\tilde{V}(r)=\sqrt{2} G_{F} \tilde{N}(r)$,

$\tilde{N}(r)$ being the reverse profile, i.e. the profile that corresponds to the interchanged positions of the 
neutrino source and detector. In the case of symmetric matter density profiles (e.g., matter of constant density), $\tilde{N}(r)=N(r)$.

An important point is that the very presence of matter (with unequal numbers of particles and antiparticles) violates $\mathrm{C}, \mathrm{CP}$ and $\mathrm{CPT}$, leading to $\mathrm{CP}$ violation in neutrino oscillations even in the absence of the fundamental $\mathrm{CP}$-violating phases $\left\{\delta_{\mathrm{CP}}\right\}$. This fake (extrinsic) CP violation may complicate the study of the fundamental (intrinsic) one.

\subsection{CP violation in matter}

Unlike in vacuum, CP violation in neutrino oscillations in matter exists even in the $2 \mathrm{f}$ case (in the case of three or more flavours, even when all $\left.\left\{\delta_{\mathrm{CP}}\right\}=0\right)$ :

$P\left(\nu_{a} \rightarrow \nu_{b}\right) \neq P\left(\bar{\nu}_{a} \rightarrow \bar{\nu}_{b}\right)$.

This is actually a well known fact - for example, the MSW effect can enhance the $\nu_{e} \leftrightarrow \nu_{\mu}$ oscillations and suppress the $\bar{\nu}_{e} \leftrightarrow \bar{\nu}_{\mu}$ ones or vice versa. Moreover, in matter the survival probabilities are not CP-invariant:

$P\left(\nu_{a} \rightarrow \nu_{a}\right) \neq P\left(\bar{\nu}_{a} \rightarrow \bar{\nu}_{a}\right)$.

To disentangle fundamental $\mathrm{CP}$ violation from the matter induced one in the LBL experiments one would need to measure the energy dependence of the oscillated signal or the signals at two baselines, which is a difficult task. The (difficult) alternatives are:

- LBL experiments at relatively low energies and moderate baselines $(E \sim 0.1-1 \mathrm{GeV}, L \sim$ $100-1000 \mathrm{~km})$ [17].

- Indirect measurements through

(A) CP-even terms $\sim \cos \delta_{\mathrm{CP}}$ [18];

(B) Area of leptonic unitarity triangle [19]. $\mathrm{CP}$ violation cannot be studied in the supernova neutrino experiments because of the experimental indistinguishability of low-energy $\nu_{\mu}$ and $\nu_{\tau}$.

\section{2. $T$ violation in matter}

Since CPT is not conserved in matter, CP and $\mathrm{T}$ violations are no longer directly connected (although some relations between them still exist [8,20]). Therefore $\mathrm{T}$ violation in neutrino oscillation in matter deserves an independent study. Its characteristic features are:
- Matter does not necessarily induce T violation (only asymmetric matter with $\tilde{N}(r) \neq N(r)$ does).

- There is no $\mathrm{T}$ violation (either fundamental or matter induced) in the $2 \mathrm{f}$ case. This is a simple consequence of unitarity. For example, for the $\left(\nu_{e}, \nu_{\mu}\right)$ system one has

$P_{e e}+P_{e \mu}=1$,

$P_{e e}+P_{\mu e}=1$,

from which $P_{e \mu}=P_{\mu e}$.

- In the $3 \mathrm{f}$ case there is only one T-odd probability difference for $\nu$ 's (and one for $\bar{\nu}$ 's), irrespective of the matter density profile:

$\Delta P_{e \mu}^{T}=\Delta P_{\mu \tau}^{T}=\Delta P_{\tau e}^{T}$.

This is a consequence of $3 \mathrm{f}$ unitarity [21].

The matter-induced $\mathrm{T}$ violation is an interesting, pure $\geq 3 \mathrm{f}$ matter effect, absent in symmetric matter (in particular, in constant-density matter). It does not vanish in the regime of complete averaging of neutrino oscillations [8]. It may fake the fundamental $\mathrm{T}$ violation and complicate its study, i.e. the extraction of $\delta_{\mathrm{CP}}$ from the experiment. The matter-induced $\mathrm{T}$ violation vanishes when either $U_{e 3}=0$ or $\Delta m_{21}^{2}=0$ (i.e., in the $2 \mathrm{f}$ limits) and so is doubly suppressed by both these small parameters. This implies that the perturbation theory can be used to obtain analytic expressions for the T-odd probability differences. The general structure of these differences is

$\Delta P_{e \mu}^{T}=\sin \delta_{\mathrm{CP}} \cdot Y+\cos \delta_{\mathrm{CP}} \cdot X$.

Here the first term $\left(\propto \sin \delta_{\mathrm{CP}}\right)$ is due to the fundamental $\mathrm{T}$ violation, whereas the second term is due to the matter-induced one. In the adiabatic approximation one finds [8] $X=J_{\text {eff }} \times$ (oscillating terms), where

$J_{\text {eff }}=s_{12} c_{12} s_{13} c_{13}^{2} s_{23} c_{23} \frac{\sin \left(2 \theta_{1}-2 \theta_{2}\right)}{\sin 2 \theta_{12}}$.

Here $\theta_{1}$ and $\theta_{2}$ are the mixing angles in matter in the (1-2) sector at the initial and final points of neutrino evolution, respectively; $\theta_{1}-\theta_{2}$ is therefore a measure of the asymmetry of the density profile. $J_{\text {eff }}$ has to be compared with the vacuum Jarlskog invariant

$J=s_{12} c_{12} s_{13} c_{13}^{2} s_{23} c_{23} \sin \delta_{\mathrm{CP}}$. 
We see that the factor $\sin \left(2 \theta_{1}-2 \theta_{2}\right) / \sin 2 \theta_{12}$ in



In an asymmetric matter, both fundamental and matter-induced $\mathrm{T}$ violations contribute to the T-odd probability differences $\Delta P_{a b}^{T}$. This may hinder the experimental determination of the fundamental CP- and T-violating phase $\delta_{\mathrm{CP}}$. In particular, in the accelerator LBL experiments one has to take into account that the Earth's density profile is not perfectly spherically symmetric. To extract the fundamental $\mathrm{T}$ violation, strictly speaking one would need to measure

$P_{\text {dir }}\left(\nu_{a} \rightarrow \nu_{b}\right)-P_{\text {rev }}\left(\nu_{b} \rightarrow \nu_{a}\right)$,

where $P_{\text {dir }}$ and $P_{\text {rev }}$ correspond to the direct and reverse matter density profiles. (An interesting point is that even the survival probabilities $P_{\mu \mu}$ and $P_{\tau \tau}$ can be used for that [22]. The $\nu_{e}$ survival probability $P_{e e}$ is an exception because in the $3 \mathrm{f}$ case it does not depend on $\delta_{\mathrm{CP}}$ [23,24]. This, however, is not true if $\nu_{\mathrm{s}}$ is present [B]).

In practical terms, it would certainly be difficult to measure the quantity in (35): It would not be easy, for example, to move CERN to Gran Sasso and the Gran Sasso Laboratory to CERN. Fortunately, this is not actually necessary - matter-induced $\mathrm{T}$ violation due to imperfect sphericity of the Earth's density distribution is very small. It cannot spoil the determination of $\delta_{\mathrm{CP}}$ if the error in $\delta_{\mathrm{CP}}$ is $>1 \%$ at $99 \%$ C.L. [8].

Can we study $\mathrm{T}$ violation in neutrino oscillations experimentally? Because of problems with the detection of $e^{ \pm}$this seems to be difficult, but probably not impossible. To study matterinduced $\mathrm{T}$ violation would be a harder task. T-odd matter effects are expected to be negligible in terrestrial experiments. They cannot be observed in the supernova neutrino oscillations because of the experimental indistinguishability of low - energy $\nu_{\mu}$ and $\nu_{\tau}$. It could, however, affect the signal from $\sim \mathrm{GeV}$ neutrinos produced in the annihilations of WIMPs inside the Sun 25].

\section{A HYMN TO $U_{e 3}$}

The leptonic mixing parameter $U_{e 3}$ plays a very special role in neutrino physics. It is of particular interest for a number of reasons.
First, it is the least known of leptonic mixing parameters: while we have (relatively small) allowed ranges for the other two mixing parameters, we only know an upper bound on $\left|U_{e 3}\right|$. Its smallness, which looks strange in the light of the fact that the other two mixing parameters, $\theta_{12}$ and $\theta_{23}$, are apparently large, remains essentially unexplained. (There are, however, some ideas which relate the smallness of $\left|U_{e 3}\right|$ to that of $\Delta m_{\odot}^{2} / \Delta m_{\text {atm }}^{2}$,26]).

The smallness of $U_{e 3}$ is likely to be the bottleneck for studying the fundamental $\mathrm{CP}$ and $\mathrm{T}$ violation effects and matter-induced $\mathrm{T}$ violation in neutrino oscillations. The same applies to the determination of the sign of $\Delta m_{31}^{2}$ in future LBL experiments, which would allow us to discriminate between the normal and inverted neutrino mass hierarchies. Therefore it would be vitally important to know how small $U_{e 3}$ actually is.

The parameter $U_{e 3}$ can be efficiently used to discriminate between various neutrino mass models [28,29. It is one of the main parameters that drives the subdominant oscillations of atmospheric neutrinos and is important for their study. It also governs the Earth matter effects on supernova neutrino oscillations.

And finally, $U_{e 3}$ apparently provides us with the only opportunity to see the "canonical" MSW effect. While matter effects can be important even in the case of large vacuum mixing angles, the most spectacular phenomenon, strong enhancement of mixing by matter, can only occur if the vacuum mixing angle is small. From what we know now, it seems that the only small leptonic mixing parameter is $U_{e 3}$.

All this makes measuring $U_{e 3}$ one of the most important problems in neutrino physics.

\section{4f OSCILLATIONS}

If the LSND experiment is correct, the oscillations interpretation of the solar, atmospheric and accelerator neutrino data would require three distinct values of $\Delta m^{2}: \Delta m_{\odot}^{2} \ll \Delta m_{\text {atm }}^{2} \ll$ $\Delta m_{\mathrm{LSND}}^{2}$. This would imply the existence of at least four light neutrino species, $\nu_{e}, \nu_{\mu}, \nu_{\tau}$ and $\nu_{s}$. A possible alternative is a strong CPT violation in the neutrino sector, leading to inequalities 
of $\Delta m^{2}$ in the neutrino and antineutrino sectors 30; I will not discuss this possibility here.

In general, the $4 \mathrm{f}$ neutrino oscillations are described by 6 mixing angles $\theta_{i j}, 3$ Dirac-type CPviolating phases and 3 values of $\Delta m_{i j}^{2}$, i.e. are quite complicated. Fortunately, there is a simplification: The data admit only 2 classes of $4 \mathrm{f}$ schemes, the so-called $(3+1)$ and $(2+2)$ schemes. In the $(3+1)$ schemes, three neutrino mass eigenstates are close to each other while the fourth one is separated from them by a large mass gap. This mass eigenstate is predominantly $\nu_{s}$ with small admixtures of the active neutrinos:

$\nu_{4} \simeq \nu_{s}+\mathcal{O}(\epsilon) \cdot\left(\nu_{e}, \nu_{\mu}, \nu_{\tau}\right), \quad \epsilon \ll 1$,

whereas $\nu_{1}, \nu_{2}$ and $\nu_{3}$ are the usual linear combinations of $\nu_{e}, \nu_{\mu}$ and $\nu_{\tau}$ plus small $(\sim \epsilon)$ admixtures of $\nu_{s}$. In this scheme the amplitude of the $\nu_{\mu} \rightarrow \nu_{e}$ oscillations at LSND is

$\sin ^{2} 2 \theta_{\mathrm{LSND}}=4\left|U_{e 4} U_{\mu 4}\right|^{2} \sim \epsilon^{4}$.

Strong upper bounds on $\left|U_{e 4}\right|$ and $\left|U_{\mu 4}\right|$ from $\bar{\nu}_{e}$ and $\nu_{\mu}$ disappearance experiments make it rather difficult to fit the LSND data in the $(3+1)$ schemes 31.

In the $(2+2)$ schemes, there are two pairs of mass eigenstates with relatively small mass squared differences, $\Delta m_{\odot}^{2}$ and $\Delta m_{\text {atm }}^{2}$, between the states within the pairs and a large separation $\left(\Delta m_{\mathrm{LSND}}^{2}\right)$ between the two pairs. The $\nu_{\mu}$ state is predominantly in the pair responsible for the atmospheric neutrino oscillations, whereas $\nu_{e}$ is mainly in the pair responsible for $\nu_{\odot}$ oscillations:

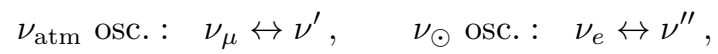

where

$\nu^{\prime} \simeq c_{\xi} \nu_{\tau}+s_{\xi} \nu_{s}+\mathcal{O}(\epsilon) \cdot \nu_{e}$,

$\nu^{\prime \prime} \simeq-s_{\xi} \nu_{\tau}+c_{\xi} \nu_{s}+\mathcal{O}(\epsilon) \cdot \nu_{\mu}$.

The amplitude of the $\nu_{\mu} \rightarrow \nu_{e}$ oscillations at LSND is

$\sin ^{2} 2 \theta_{\text {LSND }} \sim \epsilon^{2}$.

It is only of the second order in $\epsilon$ and, unlike in the $(3+1)$ case, the LSND data can be easily fitted.
However, the $(2+2)$ schemes suffer from a different problem. The fractions of $\nu_{s}$ involved in the oscillations of atmospheric and solar neutrinos must sum to unity in these schemes [32]:

$\left|\left\langle\nu_{s} \mid \nu^{\prime \prime}\right\rangle\right|^{2}+\left|\left\langle\nu_{s} \mid \nu^{\prime}\right\rangle\right|^{2} \simeq c_{\xi}^{2}+s_{\xi}^{2}=1$.

This sum rule is in conflict with the atmospheric and solar neutrino data. Indeed, the SuperKamiokande atmospheric neutrino data lead to the upper limits $\sin ^{2} \xi<0.20$ at $90 \%$ C.L. and $\sin ^{2} \xi<0.26$ at $99 \%$ C.L. [33]. At the same time, the (pre-SNO neutral current) solar neutrino data data imply $\sin ^{2} \xi>0.7$ (90\% C.L.); $\sin ^{2} \xi>0.48$ (99\% C.L.) for the LMA solution of the solar neutrino problem [34]. The recently published SNO neutral current data [14] will probably strengthen this limit. Therefore, the $(2+2)$ scenarios are also strongly disfavoured by the data.

In the $4 \mathrm{f}$ case, there may be interesting matter effects on neutrino oscillations [35]. CP violation is potentially much richer than in the $3 \mathrm{f}$ case: there are several CP-violating observables, and large CP-odd effects are possible (in general, there is no suppression due to small $\left.\Delta m_{\odot}^{2}\right)$. Also large $\mathrm{T}$ violation (both fundamental and matterinduced) can occur.

\section{CONCLUSIONS}

3f effects in solar, atmospheric, reactor and supernova neutrino oscillations and in LBL accelerator neutrino experiments may be quite important. They can lead to up to $\sim 10 \%$ corrections to the oscillation probabilities and also to specific effects, absent in the $2 \mathrm{f}$ case. The manifestations of $\geq 3$ flavours in neutrino oscillations include fundamental $\mathrm{CP}$ violation and $\mathrm{T}$ violation, matterinduced $\mathrm{T}$ violation, matter effects in $\nu_{\mu} \leftrightarrow \nu_{\tau}$ oscillations, and specific CP- and T-conserving interference terms in oscillation probabilities. The leptonic mixing parameter $U_{e 3}$ plays a very special role and its study is of great interest.

In the $4 \mathrm{f}$ case, large $\mathrm{CP}$ violation and (both fundamental and matter-induced) $\mathrm{T}$ violation effects are possible. However, if scenarios are strongly disfavoured by the data. 


\section{REFERENCES}

1. F.J. Botella, C.S. Lim and W.J. Marciano, Phys. Rev. D 35 (1987) 896.

2. V.D. Barger, K. Whisnant, S. Pakvasa and R.J. Phillips, Phys. Rev. D 22 (1980) 2718; H.W. Zaglauer and K.H. Schwarzer, Z. Phys. C 40 (1988) 273; T. Ohlsson and H. Snellman, J. Math. Phys. 41 (2000) 2768 (Erratum-ibid. 42 (2001) 2345), Phys. Lett. B 474 (2000) 153; K. Kimura, A. Takamura and H. Yokomakura, Phys. Lett. B 537 (2002) 86.

3. The CHOOZ Collaboration, M. Apollonio et al., Phys. Lett. B 420 (1998) 397; Phys. Lett. B 466 (1999) 415.

4. O. Yasuda, Acta Phys. Polon. B 30 (1999) 3089; M. Freund, M. Lindner, S.T. Petcov and A. Romanino, Nucl. Phys. B 578 (2000) 27; M. Freund, P. Huber and M. Lindner, Nucl. Phys. B 615 (2001) 331; I. Mocioiu and R. Shrock, JHEP 0111 (2001) 050.

5. A. Cervera it et al., Nucl. Phys. B 579 (2000) 17 (Erratum-ibid. B 593 (2001) 731).

6. E.Kh. Akhmedov, A. Dighe, P. Lipari and A.Yu. Smirnov, Nucl. Phys. B 542 (1999) 3.

7. O.L. Peres and A.Yu. Smirnov, Phys. Lett. B 456 (1999) 204.

8. E.Kh. Akhmedov, P. Huber, M. Lindner and T. Ohlsson, Nucl. Phys. B 608 (2001) 394.

9. O.L. Peres and A.Yu. Smirnov, Nucl. Phys. Proc. Suppl. 110 (2002) 355.

10. T.K. Kuo and J. Pantaleone, Phys. Rev. D 35 (1987) 3432; T. Ohlsson and H. Snellman, Eur. Phys. J. C 20 (2001) 507.

11. C.-S. Lim, preprint BNL-39675, 1987.

12. P.C. de Holanda and A.Yu. Smirnov, hep$\mathrm{ph} / 0205241$.

13. M.C. Gonzalez-Garcia, M. Maltoni, C. PeñaGaray and J.W.F. Valle, Phys. Rev. D 63 (2001) 033005; S.M. Bilenky, D. Nicolo and S.T. Petcov, Phys. Lett. B 538 (2002) 77; M.C. Gonzalez-Garcia and M. Maltoni, hep$\mathrm{ph} / 0202218$.

14. The SNO Collaboration, Q.R. Ahmad et al., Phys. Rev. Lett. 89 (2002) 011301.

15. C. Lunardini and A.Yu. Smirnov, Phys. Rev. D 63 (2001) 073009; C. Lunardini and A.Yu. Smirnov, Nucl. Phys. B 616 (2001) 307;
H. Minakata and H. Nunokawa, Phys. Lett. B 504 (2001) 301; V. Barger, D. Marfatia and B.P. Wood, Phys. Lett. B 532 (2002) 19.

16. E.Kh. Akhmedov, C. Lunardini and A.Yu. Smirnov, hep-ph/0204091.

17. M. Koike and J. Sato, Phys. Rev. D 61 (2000) 073012 (Erratum-ibid. D 62 (2000) 079903). H. Minakata and H. Nunokawa, Phys. Lett. B 495 (2000) 369; JHEP 0110 (2001) 001.

18. P. Lipari, Phys. Rev. D 64 (2001) 033002.

19. Y. Farzan and A.Yu. Smirnov, Phys. Rev. D 65 (2002) 113001; J.A. Aguilar-Saavedra and G.C. Branco, Phys. Rev. D 62 (2000) 096009; J. Sato, Nucl. Instr. Meth. A 472 (2000) 434.

20. H. Minakata, H. Nunokawa and S. Parke, Phys. Lett. B 537 (2002) 249.

21. P.I. Krastev and S.T. Petcov, Phys. Lett. B 205 (1988) 84.

22. P.M. Fishbane and P. Kaus, Phys. Lett. B 506 (2001) 275.

23. T.K. Kuo and J. Pantaleone, Phys. Lett. B 198 (1987) 406.

24. H. Minakata and S. Watanabe, Phys. Lett. B 468 (1999) 256.

25. A. de Gouvêa, Phys. Rev. D 63 (2001) 093003.

26. E.Kh. Akhmedov, G.C. Branco and M.N. Rebelo, Phys. Rev. Lett. 84 (2000) 3535.

27. S.F. King, hep-ph/0204360.

28. S.M. Barr and I. Dorsner, Nucl. Phys. B 585 (2000) 79.

29. M. Tanimoto, hep-ph/0106064.

30. H. Murayama and T. Yanagida, Phys. Lett. B 520 (2001) 263; G. Barenboim, L. Borissov, J. Lykken and A.Yu. Smirnov, hep-ph/0108199; G. Barenboim, J.F. Beacom, L. Borissov and B. Kayser, Phys. Lett. B 537 (2002) 227.

31. M. Maltoni, T. Schwetz and J.W.F Valle, Phys. Lett. B 518 (2001) 252; Phys. Rev. D 65 (2002) 093004.

32. O.L. Peres, A.Yu. Smirnov, Nucl. Phys. B 599 (2001) 3.

33. M. Shiozawa, these Proceedings.

34. M.C. Gonzalez-Garcia, M. Maltoni and C. Peña-Garay, Phys. Rev. D 64 (2001) 093001.

35. D. Dooling, C. Giunti, K. Kang and C.W. Kim, Phys. Rev. D 61 (2000) 073011. 